Simple voltage-controlled current source for wideband electrical bioimpedance spectroscopy: circuit dependences and limitations

This article has been downloaded from IOPscience. Please scroll down to see the full text article.

2011 Meas. Sci. Technol. 22115801

(http://iopscience.iop.org/0957-0233/22/11/115801)

View the table of contents for this issue, or go to the journal homepage for more

Download details:

IP Address: 193.11.73.8

The article was downloaded on 30/09/2011 at 12:30

Please note that terms and conditions apply. 


\title{
Simple voltage-controlled current source for wideband electrical bioimpedance spectroscopy: circuit dependences and limitations
}

\author{
F Seoane $^{1,2,4}$, R Macías ${ }^{1,3}$, R Bragós $^{3}$ and K Lindecrantz ${ }^{4}$ \\ ${ }^{1}$ School of Engineering, University of Borås, SE-501 90 Borås, Sweden \\ ${ }^{2}$ Department of Signal and Systems, Chalmers University of Technology, SE-41296 Gothenburg, \\ Sweden \\ ${ }^{3}$ Department of Electronic Engineering, Universitat Politècnica de Catalunya, Campus Nord, C-4, \\ Jordi Girona 1-3 08034, Barcelona, Spain \\ ${ }^{4}$ School of Technology and Health, KTH, Alfred Nobels Allé 10, SE-141 52 Huddinge, Sweden \\ E-mail: fernando.seoane@hb.se
}

Received 22 April 2011, in final form 2 August 2011

Published 19 September 2011

Online at stacks.iop.org/MST/22/115801

\begin{abstract}
In this work, the single Op-Amp with load-in-the-loop topology as a current source is revisited. This circuit topology was already used as a voltage-controlled current source (VCCS) in the 1960s but was left unused when the requirements for higher frequency arose among the applications of electrical bioimpedance (EBI). The aim of the authors is not only limited to show that with the currently available electronic devices it is perfectly viable to use this simple VCCS topology as a working current source for wideband spectroscopy applications of EBI, but also to identify the limitations and the role of each of the circuit components in the most important parameter of a current for wideband applications: the output impedance. The study includes the eventual presence of a stray capacitance and also an original enhancement, driving with current the VCCS. Based on the theoretical analysis and experimental measurements, an accurate model of the output impedance is provided, explaining the role of the main constitutive elements of the circuit in the source's output impedance. Using the topologies presented in this work and the proposed model, any electronic designer can easily implement a simple and efficient current source for wideband EBI spectroscopy applications, e.g. in this study, values above $150 \mathrm{k} \Omega$ at $1 \mathrm{MHz}$ have been obtained, which to the knowledge of the authors are the largest values experimentally measured and reported for a current source in EBI at this frequency.
\end{abstract}

Keywords: current source, electrical biompedance spectroscopy, operational amplifiers (Some figures in this article are in colour only in the electronic version)

\section{Introduction}

Measurement of electrical bioimpedance (EBI) is a wellestablished method for the study of various properties of body tissues. It is used for monitoring of, e.g., respiratory rate (Olsson and Victorin 1970) and tissue states such as ischaemia (Genesca et al 2005) of a patient during organ transplantation.
It is also used for different types of clinical studies, such as assessment of body composition using the BIA method (Kushner 1992).

Along with technical developments, EBI methods have gained increasing interest among physicians and researchers, and new methods based on spectral analysis of impedance are emerging. Examples of this are skin cancer screening (Aberg 


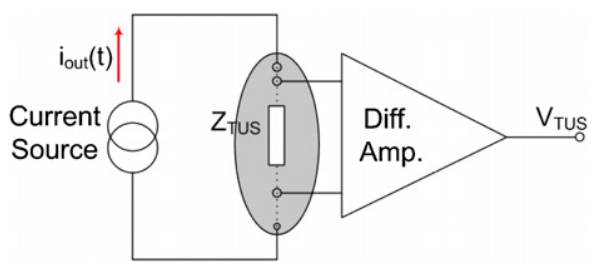

Figure 1. An ideal representation of a four-electrode voltage over current EBI measurement system.

et al 2004, Beetner et al 2003) and methods for detection of tumours (Skourou et al 2004, 2007), meningitis (Van Kreel 2001), brain cellular oedema (Lingwood et al 2002, Seoane et al 2005) and breast cancer (Assenheimer et al 2001). All these applications depend on the EBI recorded over a wide frequency range. The goal is often to obtain an impedance spectrum corresponding to the $\beta$-dispersion, i.e. between $a$ few $\mathrm{kHz}$ and up to some $\mathrm{MHz}$. Thus, EBI measurement systems with characteristics in terms of accuracy, stability and robustness at frequencies above $1 \mathrm{MHz}$ as well as at the traditionally used frequency of $50 \mathrm{kHz}$ are required.

A common extended way of measuring EBI is to inject a constant current, $I_{\text {out }}(t)$, and record the resulting voltage across the tissue of interest, $V_{\text {TUS }}$, to obtain the value of its impedance, $Z_{\text {TUS }}$; see figure 1 . For this method, the current source is an important component and its performance will influence the performance of the entire system, particularly with respect to sensitivity, frequency stability and frequency range of operation.

A key parameter of a current source is the output impedance, $Z_{\text {out }}$, as it determines both the operational frequency range and the range of load impedance in which the current source is able to maintain the constant output current, $I_{\text {out }}(t)$.

Over the years, different EBI measurement system designs have been proposed and investigated (Boone and Holder 1996), including their high-frequency performance. Consequently, several designs and approaches to the design of current sources have been implemented and studied, e.g. current conveyors (Bragos et al 1994), floating-mirrored transconductance amplifiers (Ackmann 1993), Howlandbased topologies (Jossinet et al 1994) and negative impedance converters (NICs) (Lee et al 2006, Ross et al 2003).

All these current sources operate very well at frequencies in the order of $\mathrm{kHz}$, and even at a few hundred $\mathrm{kHz}$. But with the exception of NIC-based sources, the performance degrades significantly with increasing frequency, especially the output impedance. In the NIC-based current sources, the impedance $Z_{\text {out }}$ is high at a narrow band around a single discrete frequency but it is unable to keep a large enough $Z_{\text {out }}$ over a wider frequency range. Therefore, NIC-based sources fit well for stepwise frequency sweep systems but are completely unsuitable for true spectroscopy measurements.

Advances in the development of integrated circuits have provided electronic circuit designers with reliable new wide-bandwidth Op-Amps allowing us to revisit basic structures, such as the voltage-controlled current source (VCCS) based on a simple, single Op-Amp topology in an

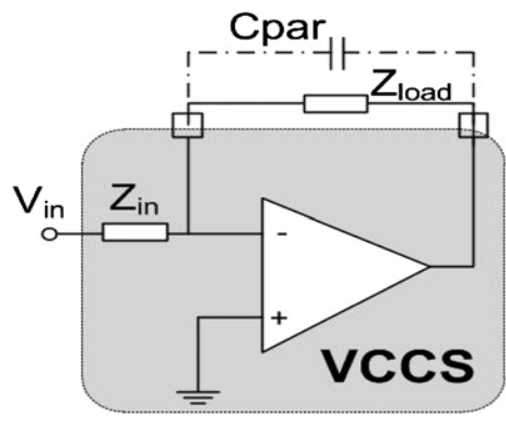

Figure 2. Load-in-the-loop current source, a traditional implementation of a VCCS based on a single Op-Amp circuit. Note that $V_{\text {in }}$ is dc free.

inverter configuration; see figure 2. In this work, first the basic equivalent model used to represent non-ideal Op-Amp circuits is validated as an accurate model to study the output impedance performance of the VCCS topology known as load-in-the-loop or floating load. Second, the dependences of the output impedance of the aforementioned topology are studied by analysing the model and through experimental test regarding the discrete circuit elements and the Op-Amp intrinsic specifications, e.g. the feedback loop resistance, the Op-Amp's differential gain, CMRR and input impedance. Finally, based on the results of the analysis, a new approach is proposed as a simple VCCS with enhanced features not only regarding the output impedance but also regarding the VCCS' transconductance.

Eventually, the output impedance at high frequency will be finally limited by the parasitic capacitance, $C_{\text {par }}$, which is, at least, due to physical connections. All current source structures will suffer this effect. Given that we would optimize the performance of the given current source by designing the parameters of the electronic devices, we will not initially consider the parasitic capacitance. The effects are shown in sections 7 and 8 .

\section{Voltage-controlled current source in electrical bioimpedance}

\subsection{General specifications of current sources}

In general terms, several parameters can be defined as linear specifications for a VCCS: input and output impedances, transconductance, i.e. the $I_{\text {out }} / V_{\text {in }}$ ratio, the transconductance's frequency response, and dc parameters such as the input bias current and output dc for zero input voltage. Input and output ranges and linearity can also be defined.

Some of these parameters, however, are non-critical in the design of a complex circuit which includes system-level calibration. The accuracy of the transconductance value and even its frequency response are systematic and could be calibrated. The input parameters will also produce systematic effects, and the adequacy of the input and output ranges should be ensured by design. The key specification of a current source connected to a variable load $Z_{L}$ is the output impedance $Z_{\text {out }}$, which also has a frequency dependence. The ratio between 


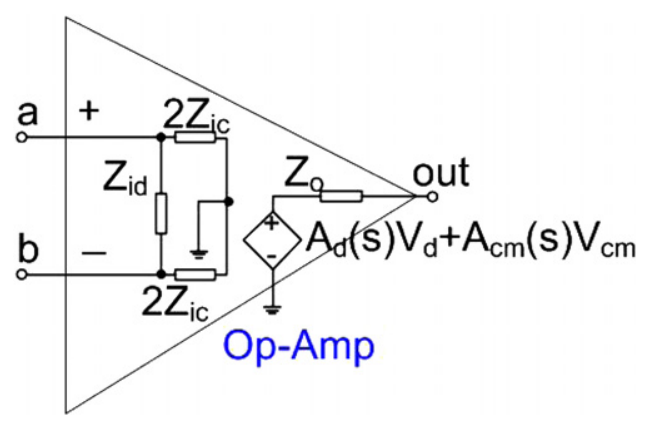

Figure 3. Equivalent model of a non-ideal Op-Amp.

$Z_{L}$ and $Z_{\text {out }}$ gives the systematic error in the current injected into the load at each frequency. If $Z_{L}$ is unknown and variable, then the error is not systematic and it should be minimized by forcing $Z_{\text {out }} \gg Z_{L}$ by design.

\subsection{Special considerations for biomedical applications}

EBI is one of the applications in which the load impedance is unknown and variable. $Z_{L}$ is composed of tissue impedances to be measured, and mainly of electrode impedances, which would present a variation that in some cases could be larger than the impedance under measurement. Thus, $Z_{\text {out }}$ should be much higher than these impedance values or, at least, than their variations. This condition could easily be achieved at low frequency but not above $100 \mathrm{kHz}$, where $Z_{\text {out }}$ is dominated by the output capacitance.

The alternative of applying voltage instead of injecting current has several drawbacks. The current is not intrinsically limited, and the safety is also not ensured. The limitation imposed by the standard IEC-60601 is defined in terms of current. The current measurement circuits, which are mandatory in this case, could have the same load-dependent errors as current sources, and the use of a variable current could induce nonlinear effects.

Another condition that arises when applying a current source to a living tissue is the need of decoupling the residual dc. This is usually done by placing capacitors in the injecting leads in series with the load preventing any dc from flowing through the load, as in figure 2. A dc path should be provided to these currents. A straightforward method is the use of a resistor in parallel with the current source, $R_{f}$, but this will result in a reduction of $Z_{\text {out }}$. The alternative of using a dc-feedback circuit involves a circuit complexity that can be avoided by ensuring a very low value for this dc, which allows the usage of a resistor $R_{f}$ with a large resistance.

\section{Materials and methods}

\subsection{Circuit analysis}

The current source circuits studied in this work have been analysed by using equivalent circuit models for the non-ideal Op-Amp as in figure 3. For each circuit, an equivalent model of the VCCS has been created and the expression for $Z_{\text {out }}$ of the model has been found.
Following Jaeger and Blalock (2004), the circuit input has been analysed and $Z_{\text {ic }}$, the common-mode input impedance in (1), and $Z_{\mathrm{id}}$, the differential-mode input impedance (2), have been calculated, using the values provided by the manufacturer of the Op-Amp circuits, selected for each of the studied circuit topologies:

$$
\begin{aligned}
& Z_{\mathrm{ic}}=R_{\mathrm{ic}} \|\left(\frac{1}{\mathrm{j} \omega C_{\mathrm{ic}}}\right) \\
& Z_{\mathrm{id}}=R_{\mathrm{id}} \|\left(\frac{1}{\mathrm{j} \omega C_{\mathrm{id}}}\right) .
\end{aligned}
$$

The resulting expressions for the equivalent $Z_{\text {in }}$ and $Z_{\text {out }}$ have been developed, and their values have been calculated for the same set of values of the discrete components and the integrated circuit specifications of the Op-Amps that were used in each implementation, as described below.

In the analysis of $Z_{\text {out }}$, we have considered the gain of the Op-Amp as the relationship between its output and input:

$$
\begin{aligned}
V_{\mathrm{out}}(s) & =A_{d}(s) \times V_{d}(s)+A_{\mathrm{cm}}(s) \times V_{\mathrm{cm}}(s) \\
& =A_{d}(s) \times\left(V_{d}(s)+V_{\mathrm{cm}}(s) / \operatorname{CMRR}(s)\right),
\end{aligned}
$$

where $A_{d}$ is the differential mode gain, $A_{\mathrm{cm}}$ is the common mode gain of the Op-Amp, $V_{d}$ and $V_{\mathrm{cm}}$ are the differential- and common-mode input voltages of the Op-Amp, respectively, and $\operatorname{CMRR}(s)$ is the ratio between both gains.

\subsection{Physical implementation}

To compare the model results with experimental results from physical realizations, the VCCS circuits were implemented on a single PCB using as many surface mount devices (SMD) as possible and a link connector to switch each configuration. Furthermore, the following active components were used.

- The integrated circuits LMH6654 and OP07D in a SOIC package for the implementation of the load-in-the-loop VCCS.

- The current feedback Op-Amp integrated circuit AD844 in a SOIC encapsulation has been used for driving the load-in-the-loop VCCS in a second topology.

- The differential amplifier AD8130 in a SOIC package for measuring the floating voltage from which the output impedance is derived.

\subsection{Output impedance analysis}

Equivalent models for the output of each VCCS circuit have been obtained together with analytical expression for the corresponding output impedance, $Z_{\text {out }}$. The dependence of $Z_{\text {out }}$ with respect to the values of the discrete components and the intrinsic parameters of the active components has been studied by using the obtained model and the expression for $Z_{\text {out }}$. 


\section{Test and output impedance measurements}

Different specific measurement circuits have been built implementing the VCCS under study for test and validation of the model: the load-in-the-loop VCCS and the proposed enhancement. The output impedance of the implemented current sources has been measured with the impedance analyser LCR HP4192A in gain/phase measurement mode, applying the technique used by Bertemes-Filho et al (2000). The obtained measurement results have been compared with the calculated values resulting from the output impedance analysis.

\subsection{Op-Amp's parameters' dependence}

For this experiment, the VCCS topology with load-in-the-loop has been implemented with two different Op-Amp circuits: the OP07 and the LMH6654. The first is a general purpose OpAmp circuit with the dominant pole of the differential gain below $10 \mathrm{kHz}$, while the second is a wideband Op-Amp with the dominant pole at $125 \mathrm{kHz}$. This experiment pursues the validation of the obtained equivalent model in the frequency domain, by studying the influence of the differential gain of the Op-Amp on the obtained output impedance.

\subsection{Influence of the passive components of the VCCS circuit}

In this experiment, output impedance measurements have been performed on a load-in-the-loop VCCS for different values of $R_{\text {in }}$ and the feedback protection resistor $R_{f}$. This experiment is to validate that the values of the passive components of the VCCS circuit influence the output impedance as predicted by the obtained equivalent model.

\subsection{Current driven load-in-the-loop}

This enhancement consists of a VCCS connected in cascade with a load-in-the-loop circuit topology acting as a currentcontrolled current source (CCCS); see figure 6. The first VCCS is a current conveyor whose current output drives the input of the load-in-the-loop CCCS. From an output impedance perspective, this is practically equivalent to adding to the value of the input resistor $R_{\text {in }}$ present in the load-in-theloop topology, the output impedance of the $T_{z}$ terminal, which is higher. This change also breaks the dependence between the transconductance value and the output impedance, which was inherent to the first structure.

\section{Single Op-Amp VCCS}

\subsection{General considerations}

Figure 4 illustrates one of the first examples of a single Op-Amp circuit VCCS with the working load in the loop (Sheingold 1966), which is known as load-in-the-loop current source. With an ideal Op-Amp, the characteristics of the circuit are determined solely by the passive components, but in real implementations the performance of the circuit at higher frequencies will depend largely on the non-ideal properties and the specifications of the Op-Amp as well.

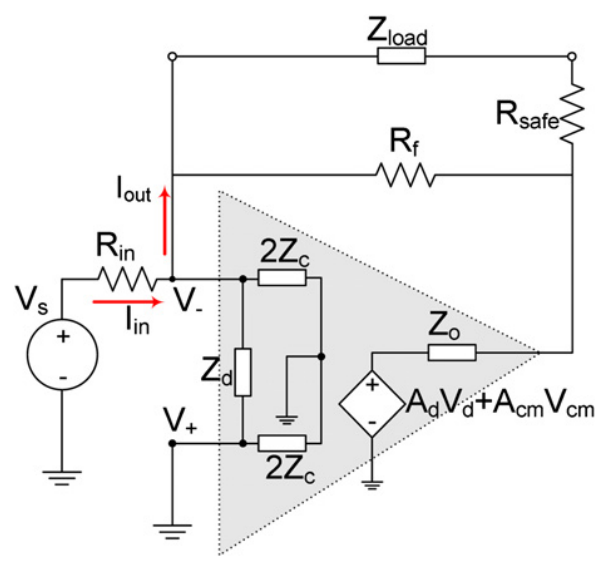

Figure 4. The circuit under study, the load-in-the-loop current source. In practice, the load is free from dc due to capacitors in series with the injecting leads and the electrodes, not shown in the figure.

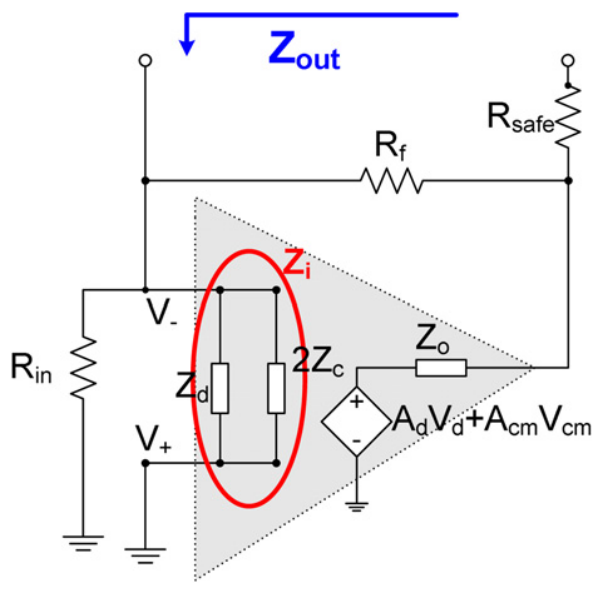

Figure 5. Equivalent circuit used to calculate the output impedance of the VCCS.

\subsection{Circuit analysis results}

5.2.1. Output current. The VCCS's output current, $I_{\text {out }}$, is given by the ratio between $V_{S}$ and $R_{\text {in }}$; see (4) and figure 4. $R_{\text {in }}$ is then the transconductance of the current source:

$$
I_{\text {out }} \approx I_{\text {in }}=\frac{V_{S}}{R_{\text {in }}} .
$$

5.2.2. Output impedance. The output impedance $Z_{\text {out }}$ of the VCCS is found from the equivalent circuit in figure 5. The analytical expression for the Op-Amp's input impedance is written as

$$
Z_{i}=2 Z_{\text {ic }} \| Z_{\text {id }}
$$

and (6) contains the final expression for the corresponding output impedance of the VCCS, $Z_{\text {out }}$ :

$$
Z_{\text {out }}=R_{\text {safe }}+R_{f} \|\left(Z_{o}+\left(R_{\text {in }} \| Z_{i}\right) \text { opz }(s)\right),
$$

where the operational amplifier impedance factor $\operatorname{opz}(s)$ is defined as

$$
\operatorname{opz}(s)=\left(A_{d}(s)+1\right)\left(1-\frac{1}{2 \operatorname{CMRR}(s)}\right) .
$$


Table 1. Values used for calculating the $Z_{\text {out }}$ magnitude.

\begin{tabular}{|c|c|c|c|c|c|}
\hline \multirow{2}{*}{$\frac{\text { Symbol }}{Z_{d}}$} & \multirow{2}{*}{$\begin{array}{l}\text { Expression } \\
R_{\mathrm{id}} / / C_{\mathrm{id}}\end{array}$} & \multicolumn{2}{|c|}{ Values for LMH6654 } & \multicolumn{2}{|l|}{ Values for OP07 } \\
\hline & & $R_{\mathrm{id}}=20 \mathrm{k} \Omega$ & $C_{\mathrm{id}}=0.55 \mathrm{pF}$ & $R_{\mathrm{id}}=50 \mathrm{M} \Omega$ & \\
\hline$Z_{\text {ic }}$ & $R_{\mathrm{cm}} / / C_{\mathrm{cm}}$ & $R_{\mathrm{cm}}=4 \mathrm{M} \Omega$ & $C_{\mathrm{cm}}=0.9 \mathrm{pF}$ & $R_{\mathrm{cm}}=160 \mathrm{G} \Omega$ & \\
\hline$A_{d}(s)$ & $A_{d 0} /\left(1+\left(s / \omega_{d}\right)\right)$ & $A_{d 0}=80 \mathrm{~dB}$ & $\omega_{d}=2 \pi \times 125 \mathrm{kHz}$ & $A_{d 0}=113 \mathrm{~dB}$ & $\omega_{d}=2 \pi \times 4 \mathrm{~Hz}$ \\
\hline $\operatorname{CMRR}(s)$ & $\mathrm{CMRR}_{0} /\left(1+\left(s / \omega_{\mathrm{cm}}\right)\right)$ & $\mathrm{CMRR}_{0}=100 \mathrm{~dB}$ & $\omega_{\mathrm{cm}}=2 \pi \times 9 \mathrm{kHz}$ & $\mathrm{CMRR}_{0}=127 \mathrm{~dB}$ & $\omega_{\mathrm{cm}}=2 \pi \times 30 \mathrm{~Hz}$ \\
\hline$Z_{o}$ & & $80 \Omega$ & & $60 \Omega$ & \\
\hline$R_{\text {in }}$ & & $6.2 \mathrm{k} \Omega$ & & $6.2 \mathrm{k} \Omega$ & \\
\hline$R_{\text {safe }}$ & & $390 \Omega$ & & $390 \Omega$ & \\
\hline$R_{f}$ & & $390 \mathrm{k} \Omega$ & & $390 \mathrm{k} \Omega$ & \\
\hline
\end{tabular}

Note. $\mathrm{CMRR}_{0}$ and $A_{d 0}$ of LMH6654 have been extracted from the plots of CMRR versus frequency and the open loop gain versus frequency included in the datasheet.

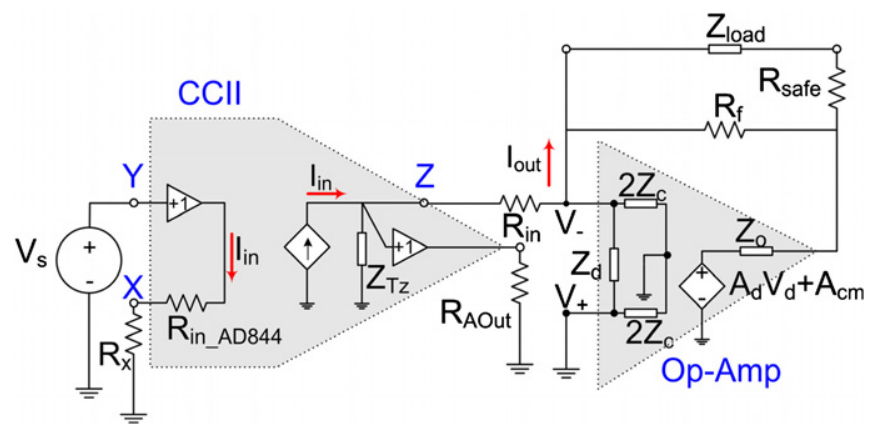

Figure 6. Current source proposed. A load-in-the-loop VCCS circuit driven by a current conveyor.

The magnitude of the output impedance has been calculated with the values from table 1 , and the frequency dependence is plotted in section 7. Note that the frequency response of the differential gain $A_{d}(s)$ has been simplified to a single-pole expression. In the first approach, the dominating parameters in expression (6) are the open loop gain $A_{d}$ at a given frequency and the current determining resistor $R_{\text {in }}$. This last component value cannot be arbitrarily increased, given that this implies increasing also the input control voltage $V_{S}$. Its practical value is limited to a few $\mathrm{k} \Omega$ for control voltages around $1 \mathrm{~V}$ and output currents in the range of $100 \mu \mathrm{A}-1 \mathrm{~mA}$.

\section{Load-in-the-loop driven by the current}

\subsection{General considerations}

The influence of the equivalent impedance formed by the OpAmp's input impedance, $Z_{i}$, in parallel with the impedance at the inverting input of the Op-Amp, $R_{\text {in }}$, on the overall output impedance of the whole VCCS circuit, $Z_{\text {out }}$, is clearly seen in (6). In an attempt to increase the value of this equivalent impedance, we have modified the original load-in-the-loop VCCS topology from figure 4, replacing the Thevenin source $V_{S}$ by a Norton source and thereby increasing the equivalent $R_{\text {in }}$. The implemented VCCS is driven by a primary VCCS using AD844 as a current conveyor (CCII) in the previous stage, as in figure 6 . The circuit topology is similar to a cascode structure: the first stage converting voltage to current with low output voltage excursion followed by a current buffer. This modification also tries to add a degree of freedom in the design by removing the VCCS transconductance determining resistor, $R_{x}$ in figure 6 , from the VCCS $Z_{\text {out }}$ expression.

\subsection{Circuit analysis results}

Following the circuit equivalent's analytical approach and experimental approach explained in the previous section, this topology has been studied elsewhere by Seoane et al (2006) and the analytical expression for the output impedance is obtained; see

$$
Z_{\text {out }}=R_{\text {safe }}+R_{f} \|\left(Z_{o}+\left(\left(R_{\text {in }}+Z_{T_{z}}\right) \| Z_{i}\right) \text { opz }(s)\right),
$$

where $Z_{i}$ is given in (5), $Z_{T_{z}}$ is the output impedance of the terminal $T_{z}$, as indicated in figure 6 , and its expression and values are given in

$$
Z_{T_{Z}}=R_{t} \|\left(\frac{1}{\mathrm{j} \omega C_{t}}\right)=\frac{3 \mathrm{M} \Omega \| 1}{\mathrm{j} \omega 4.5 \mathrm{pF}} .
$$

\section{Results}

\subsection{Influences of the Op-Amp's intrinsic parameters}

Figure 7 shows the plots for the output impedance measurements obtained with the load-in-the-loop VCCS topology for both Op-Amp circuits: LMH6654 and OP07. The measurements are represented with circle markers and square markers for LMH6654 and OP07, respectively. For validation and comparison purposes, figure 7 also shows the output impedance obtained from the model calculations, represented with a continuous trace for LMH6654 and a dashed trace for OP07. Both sets of curves show close agreement between the measured and calculated impedance values, except for the effect of the parasitic capacitance at high frequency, which is commented on in section 8 . The difference in the output impedance of both circuits at high frequency is clearly exhibited. Also at low frequencies, a slightly larger $Z_{\text {out }}$ value for the OP07 circuit is obtained.

\subsection{Influence of the values of the passive components on the output impedance}

The influence of the resistance $R_{\text {in }}$ at the input of the VCCS and the feedback protection resistor $R_{f}$ on the output impedance $Z_{\text {out }}$ is shown in figures 8 and 9 , respectively. The figures 


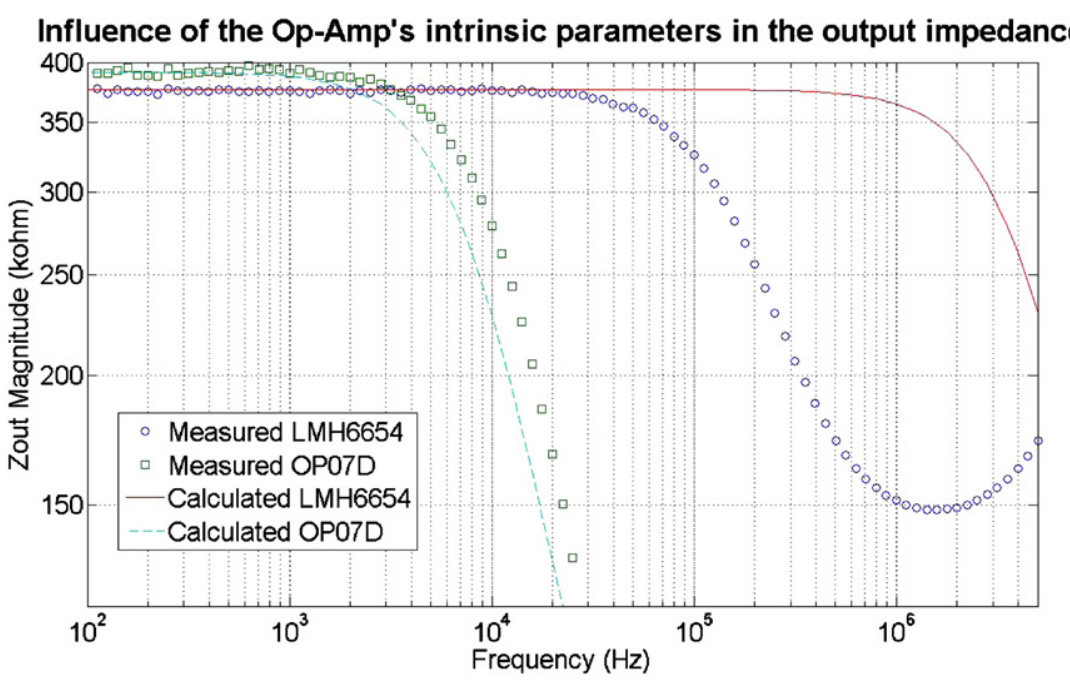

Figure 7. Limitation of the frequency range depending on the Op-Amp. As the Op-Amp OP07D has smaller bandwidth than the cut-off frequency, the parasitic capacitance does not limit the frequency range. On the other hand, using the Op-Amp LMH6654, the parasitic capacitance limits the frequency range. The value for $R_{f}$ is $390 \mathrm{k} \Omega$ and for $R_{\text {in }}$ is $6.2 \mathrm{k} \Omega$.
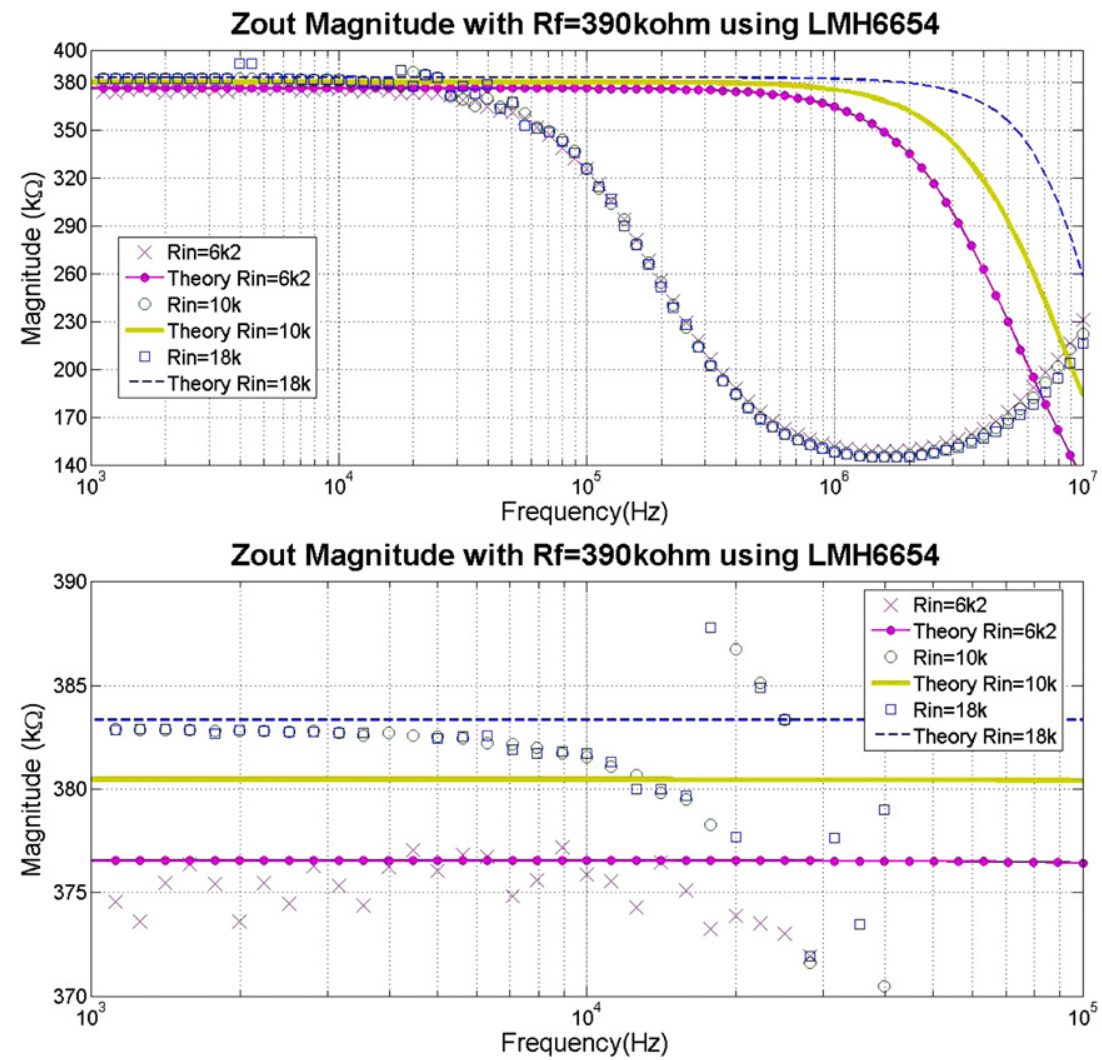

Figure 8. Top: $Z_{\text {out }}$, the magnitude of the load-in-the-loop current source using a $390 \mathrm{k} \Omega$ feedback protection resistor and several values of the input resistor: $6.2 \mathrm{k} \Omega$ (cross marker and thin continuous trace with a point marker), $10 \mathrm{k} \Omega$ (circle marker and thick continuous trace) and $18 \mathrm{k} \Omega$ (square marker and dashed trace). Bottom: the same plot enlarged to show the role of the input resistor at low frequencies, from 1 to $100 \mathrm{kHz}$.

show both experimental and calculated values, markers and traces, respectively. Therefore, it is possible to observe in each figure the close agreement at low frequencies between the values obtained experimentally and the calculated ones. The disagreement between the calculated and the measured values begins at frequencies above $25 \mathrm{kHz}$. While at low frequencies and in accordance with the calculated values there are certain differences in the magnitude of the output impedance, at frequencies above $70 \mathrm{kHz}$ all the measured values match one another, deviating in all cases from the calculated values, due again to the effect of the parasitic capacitance as will be discussed later. 


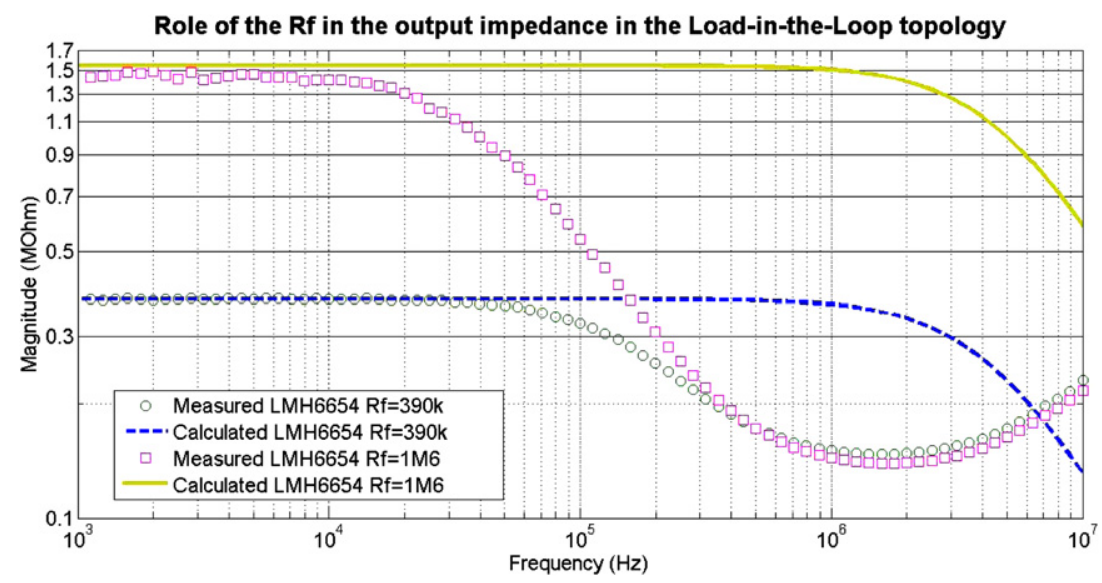

Figure 9. $Z_{\text {out }}$, the magnitude of the load-in-the-loop current source using a fixed value of the input resistor, $6.2 \mathrm{k} \Omega$, and changing the value of the feedback resistor: $390 \mathrm{k} \Omega$ (circle marker and dashed trace) and 1.6 M $\Omega$ (square marker and continuous trace).

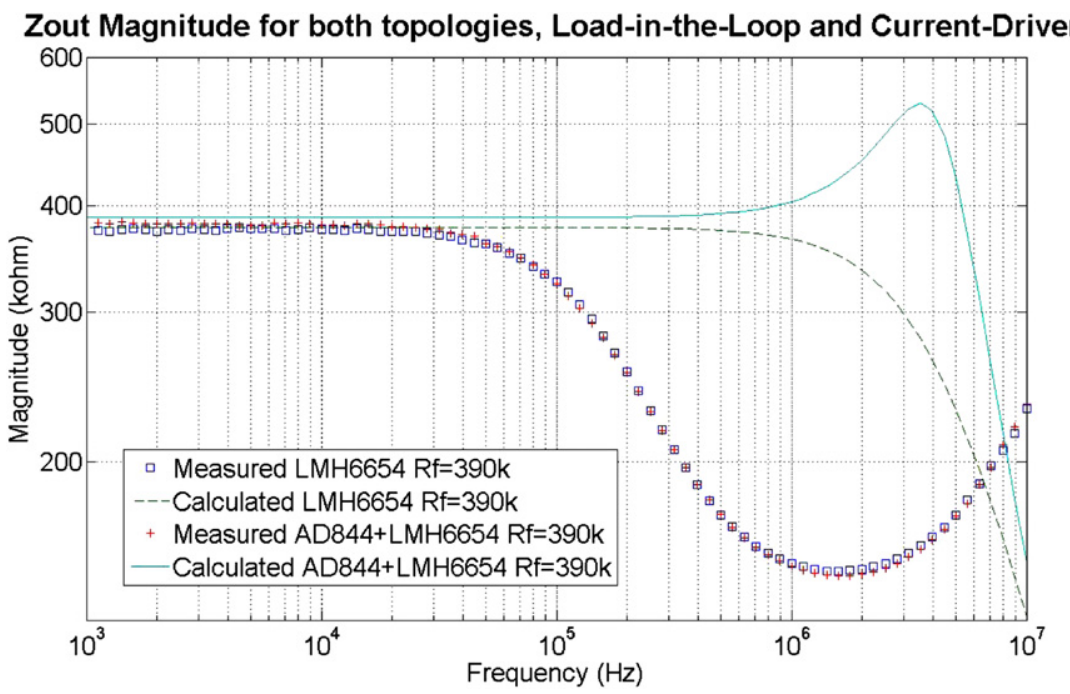

Figure 10. $Z_{\text {out }}$, the magnitude of the VCCS topologies using an input resistor of $6.2 \mathrm{k} \Omega$ and a feedback resistor of $390 \mathrm{k} \Omega$.

All the values used in the calculations as well as the values selected for the physical implementation are the same, and are indicated in table 1.

\subsection{Current-driven load-in-the-loop}

Figure 10 illustrates the measurements and calculations for the output impedance of the current-driven VCCS comparing the results with measurements for the original load-in-the-loop circuit. Figure 10 shows similar behaviour at all frequencies for the output impedance measured on both circuits: square marker for load-in-the-loop and cross marker for the currentdriven VCCS. The obtained experimental measurements, indicated with the continuous trace for the current-driven VCCS and the dashed trace for the original topology, differ significantly from the theoretical output impedance at $50 \mathrm{kHz}$ and above.

\subsection{Influence of the parasitic capacitance at the output}

The output impedance obtained from measurements for the load-in-the-loop topology and the VCCS driven by the current are plotted in figures 11 and 12, respectively. In both plots, it is possible to see that there is a dominant pole in the measurements (squares), which only fit with the calculated responses (continuous trace) if a capacitor of $2.7 \mathrm{pF}$ is placed in parallel with the load in the model to simulate the stray capacitance $C_{\mathrm{par}}$. To corroborate this hypothesis, an additional capacitor of $2.7 \mathrm{pF}$ is placed both in the circuit and in the model, obtaining the responses represented by circles and dashed lines, respectively, with the dominant pole at lower frequencies. The agreement is valid only at low and medium frequencies $(<200 \mathrm{kHz})$. Above this range, probably higher order effects in the real amplifier behaviour or even in the measurement set-up break the monotonic tendency of the measured impedances. In any case, the effect that causes the main decay in the performance of the current source is the stray capacitance $C_{\mathrm{par}}$.

\section{Discussion}

This circuit topology and the equivalent model used for the analysis are very simple and have few components and 


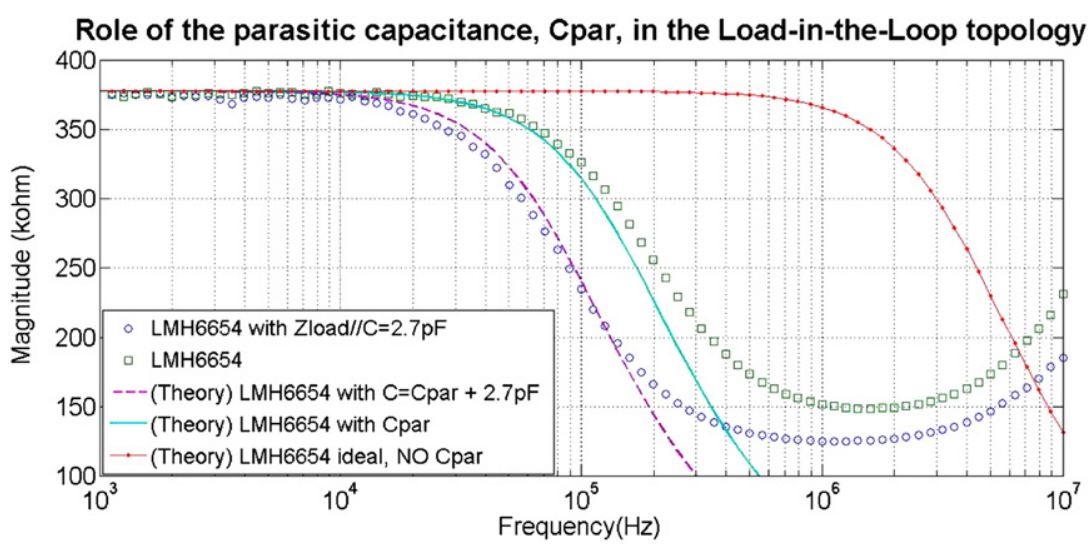

Figure 11. Plots of the output impedance, measurements and calculations for the load-in-the-loop topology, under ideal conditions and including parasitic capacitance.

Role of the parasitic capacitance, Cpar, in the Current-Driven VCCS

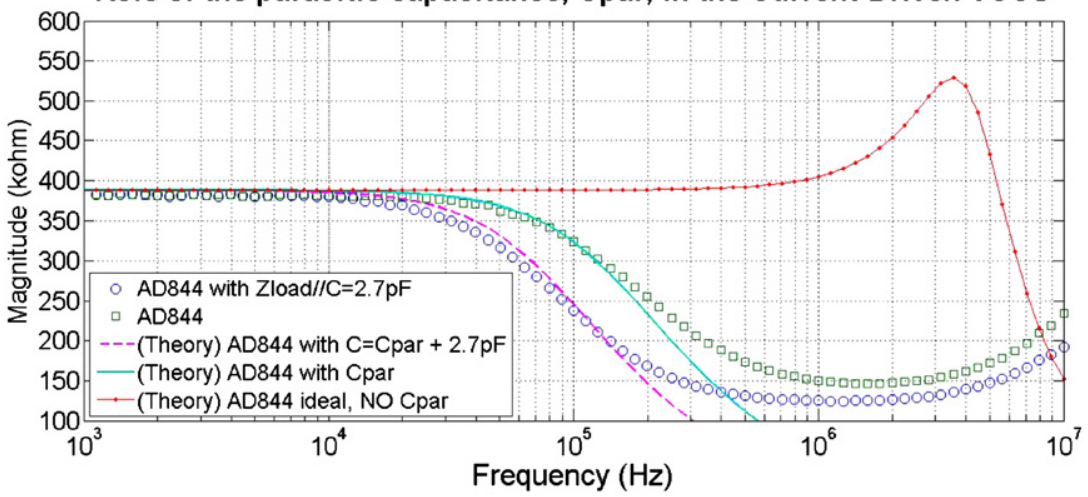

Figure 12. Plots of the output impedance, measurements and calculations for the VCCS driven by current, under ideal conditions and including parasitic capacitance.

parameters. From the experimental measurements, we have been able to confirm that every element of the circuit and the equivalent model, both the resistors and the IC's equivalent model parameters, influence the output impedance of the current source.

\subsection{Passive components}

8.1.1. Regarding $R_{\mathrm{in}}$. As shown in figure 8, increasing the value of $R_{\text {in }}$ increases the value of $Z_{\text {out }}$, within the obvious limitations associated with the parallel connection to the rest of elements. The agreement between the experimental and the calculated values plotted in figure 8 validates the use of the equivalent model regarding the contribution of $R_{\text {in }}$ to the total output impedance. $R_{\text {in }}$ also sets the transconductance of the source and this fact must be considered when selecting its value.

8.1.2. Regarding $R_{f}$. The feedback protection resistor is in parallel connection to the output of the current source, acting as a current divider of the output current. Therefore, the smaller the value of $R_{f}$, the smaller the output impedance. $R_{f}$ ensures the negative feedback of the Op-Amp keeping the current source functioning as such at all times even when the load is disconnected, which might not be a serious problem in practice after all. In addition, $R_{f}$ also provides a current divider for ac due to its self-capacitance (Broudy and Levinstein 1954). Such a capacitance might be very small, below $\mathrm{pF}$, but it adds to other parasitic capacitances associated with the output. Since the presence of this resistor decreases the value of the output impedance of the source, all these issues together with the Boella effect must be considered during the design phase to select the appropriate resistor value, geometry and material. Again, the experimental and simulated results plotted in figure 9 validate the use of the equivalent circuit regarding the contribution of $R_{f}$ to the total output impedance of the VCCS.

\subsection{Operational amplifier}

The more important parameters of the Op-Amp circuit for the total output impedance of this type of VCCS circuit are the input impedance $Z_{i}$, the differential gain $A_{d}(s)$ and the common-mode rejection ratio $\operatorname{CMRR}(s)$. The combination of the last two defines the opz factor; see (7).

The 'opz' factor not only multiplies and propagates to the output impedance $Z_{\text {out }}$ the input of the Op-Amp circuit, but also any other impedance at the input, therefore attenuating the 
effect of any capacitance associated with the input. Among the Op-Amp's parameters, the main factor contributing to the output impedance of the VCCS is the differential gain $A_{d}(s)$; therefore, its maximum value, $A_{d 0}$ in a single-pole system, as well as its frequency response set by the frequency of the dominant pole is critical for the output impedance of the VCCS circuit, especially at high frequencies. Again, the validity of the equivalent circuit for modelling the contribution of the OpAmp circuit's intrinsic parameters is supported by the output impedance plotted in figure 7.

\subsection{Current drive}

According to the analysis of the proposed equivalent model of both VCCS circuits, to drive with current source the loadin-the-loop topology would produce an output impedance on the VCCS larger than that with the original circuit at high frequencies. The results indicate that the expected increase does not occur and instead, the output impedance of VCCS remains the same. The reason behind this unexpected result resides in the presence of a stray capacitance in parallel with the measurement load. This way, the stray capacitance impedes the achievement of any larger output impedance at high frequencies. The improvement introduced by driving the original VCCS with a current source is to break the dependence between the value of the output impedance and the transconductance of the load-in-the-loop topology indicated in equations (4) and (6). This way, the output current is set at the input of the current conveyor completely independent of the values of the components used in the load-in-the-loop circuit, then adding a degree of freedom. Nevertheless, with the current devices' parameter values, this effect could not be observed clearly.

\subsection{Output parasitic capacitance}

It is easy to realize from figures 7, 9 and 12 that the impedance obtained with the model only matches the measured impedance at low frequencies. Only when adding a stray capacitance in parallel with the output is it possible to obtain reasonable agreement between the model and the experimental measurements. At low frequencies, the stray capacitance $C_{\mathrm{par}}$ does not influence the output impedance of the VCCS as can be observed in figure 7 , where the agreement between the experimental and calculated output impedance for the Op-Amp circuit OP07 is very good. At frequencies above $100 \mathrm{kHz}$, the effects of the circuit and parasitic capacitances are not negligible. The measurements plotted in figures 810 clearly show how these capacitances dominate at high frequencies producing exactly the same output impedance regardless of the implemented VCCS topology or the value of the used passive components.

The measurements and calculations performed with the added capacitance shown in figures 11 and 12 confirm that the decreasing value of the output impedance is associated with a stray capacitance of approximately $2.7 \mathrm{pF}$.

Careful component selection together with the right circuit implementation, i.e. using only SMD components, using short connections, avoiding parallel tracks, etc, would probably

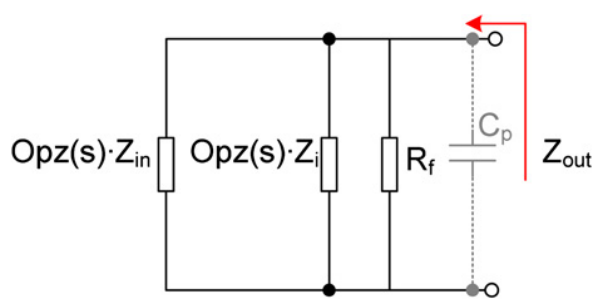

Figure 13. General equivalent circuit for the output impedance for both topologies and considering the effect of the parasitic capacitance.

reduce the effect of the parasitic capacitances significantly. It is evident that a microelectronic implementation as integrated circuit will provide the best performance and will be the most robust option to minimize parasitic capacitances. Nevertheless, a few $\mathrm{pF}$ are unavoidable in the practical connection of the integrated circuit with the printed circuit board, connectors and electrodes.

\subsection{Frequency effects}

The initial aim of this paper was to relate the performance of a given current source topology to the parameters of the devices used in its implementation, in order to provide an aid for designers and to demonstrate that a very simple structure can have a very good performance. The results show that at low frequencies the frequency response of the output impedance is dominated by effects predicted by the model, while at high frequencies the determining factor is the unavoidable output parasitic capacitance. The validation has been done only with two structures, but any other current source topology will also suffer this effect and most commercial impedance analysers have high errors at high frequencies (Bogonez-Franco et al 2009). The load-in-the-loop structure has the advantage of being very simple and, with modern operational amplifiers, the output impedance frequency response that can be obtained is enough for bioimpedance applications and is limited at high frequencies by a physical cause that is shared with other structures.

\section{Conclusions}

From the comparison between the experimental measurements and the calculated impedance, it can be concluded that through the equivalent circuit model used to analyse the output impedance of the topologies, all the circuit dependences have been confirmed. Once the stray capacitance has been included into the model, the agreement between theoretical and experimental values improves. The simple model used and the stray capacitance allow us to produce an effective simplified model for the output impedance of the VCCS circuits studied in this work based on the passive components and the intrinsic parameters of the Op-Amp and the CCII.

The obtained general equivalent circuit model depicted in figure 13 is very straightforward and it is composed of four impedances in parallel: three from the circuit elements and one parasitic. $Z_{i}$ is the input impedance of the VCCS considering the Op-Amp ideal, $Z_{\text {in }}$ is the equivalent input 
impedance of the Op-Amp from the inverting input to ground, $R_{f}$ is the impedance in the feedback loop, $C_{p}$ is the parasitic capacitance associated with the output and opz $(s)$ is the OpAmp impedance factor as defined in (7). $Z_{\text {in }}$ will be the resistance at the input $R_{\text {in }}$ for the original load-in-the-loop and $R_{\text {in }}+\mathrm{Z}_{T Z}$ for the current-driven version. Note that the values of $Z_{o}$ and $R_{\text {safe }}$ have been considered negligible.

The obtained equivalent circuit model, which relates the VCCS main parameter $Z_{\text {out }}$ to the Op-Amp parameters and the passive circuit elements, is a useful tool that enables the designer to select the most adequate commercial Op-Amp and the values for the input and feedback resistors for a given VCCS specification.

It is noteworthy to indicate that the experimental results obtained in this study have proven that is possible to obtain a large output impedance with a simple Op-Ampbased VCCS at high frequencies, e.g. $150 \mathrm{k} \Omega$ at $1 \mathrm{MHz}$. Currently available bioimpedance spectrometers such as SFB7 (Impedimed, Brisbane), BCM (Fresenius Medical Care Deustchland, Germany) and Z-Métrix ${ }^{\circledR}\left(\right.$ BioparmHom $^{\odot}$, France) operate with an upper frequency limit of $1 \mathrm{MHz}$ for body composition assessment. Thus, any of the load-in-theloop topologies presented in this work exhibiting such an output impedance in the frequency range near $1 \mathrm{MHz}$ could be used in such an application.

Since the load-in-the-loop topology is that simple, there is an important dependence between the transconductance determining resistor $R_{\mathrm{in}}$ and the output impedance. Driving with current, the VCCS should remove such significant limitation. Unfortunately, due to the stray capacitance, driving with current did not increase the output impedance as expected, and such a capacitance is the true limiting factor to obtaining large output impedance at high frequencies. In the frequency range of the order of $1 \mathrm{MHz}$, however, the effect of parasitic capacitances is decisive for the performance of the current source. In this study, the stray capacitance cancels the benefit that would have been produced by driving the input with current. In order to minimize their destructive effects, parasitic capacitances must be taken into account at the earliest stage of the circuit design, trying to minimize their value through the use of distributed or semi-distributed system architectures if possible.

After all the measurements and validation test, the authors can conclude that current sources for electrical bioimpedance measurements with appropriate performance for wideband spectroscopy applications can be implemented by using a single Op-Amp circuit. There are basically three requirements for such Op-Amp circuits: large differential input impedance, low bias currents to allow high $R_{f}$ and, most importantly, an open-loop gain with the first pole in its frequency response as high in frequency as possible. Applying these design rules together with the general equivalent circuit model presented for the VCCS wideband spectroscopy, bioimpedance applications can be implemented.

\section{Acknowledgments}

The authors would like to thank Dr Ants Silberberg and Dr Roger Malmberg for their helpful comments on the performance of Op-Amp circuits and the effect of parasitic capacitances.

\section{References}

Aberg P, Nicander I, Hansson J, Geladi P, Holmgren U and Ollmar S 2004 Skin cancer identification using multifrequency electrical impedance-a potential screening tool IEEE Trans. Bio. Med. Eng. 51 2097-102

Ackmann J J 1993 Complex bioelectric impedance measurement system for the frequency range from $5 \mathrm{~Hz}$ to $1 \mathrm{MHz} A n n$. Biomed. Eng. 21 135-46

Assenheimer $\mathrm{M}$ et al 2001 The T-SCAN ${ }^{\mathrm{TM}}$ technology: electrical impedance as a diagnostic tool for breast cancer detection Physiol. Meas. 22 1-8

Beetner D G, Kapoor S, Manjunath S, Zhou X and Stoecker W V 2003 Differentiation among basal cell carcinoma, benign lesions, and normal skin using electric impedance IEEE Trans. Biomed. Eng. 50 1020-5

Bertemes-Filho P, Brown B H and Wilson A J 2000 A comparison of modified Howland circuits as current generators with current mirror type circuits Physiol. Meas. 21 1-6

Bogonez-Franco P, Nescolarde L, Bragos R, Rosell-Ferrer J and Yandiola I 2009 Measurement errors in multifrequency bioelectrical impedance analyzers with and without impedance electrode mismatch Physiol. Meas. 30 573-87

Boone K G and Holder D S 1996 Current approaches to analogue instrumentation design in electrical impedance tomography Physiol. Meas. 17 229-47

Bragos R, Rosell J and Riu P 1994 A wide-band AC-coupled current source for electrical impedance tomography Physiol. Meas. 15 (Suppl. 2a) A91-99

Broudy R and Levinstein H 1954 High-frequency resistance of thin films Phys. Rev. 94285

Genesca $\mathrm{M}$ et al 2005 Electrical bioimpedance measurement during hypothermic rat kidney preservation for assessing ischemic injury Biosens. Bioelectron. 20 1866-71

Jaeger R C and Blalock T N 2004 Microelectronic Circuit Design 2nd edn (Singapore: McGraw-Hill) pp 752

Jossinet J, Tourtel C and Jarry R 1994 Active current electrodes for in vivo electrical impedance tomography Physiol. Meas. 15 (Suppl 2a) A83-90

Kushner R F 1992 Bioelectrical impedance analysis: a review of principles and applications J. Am. Coll. Nutr. 11 199-209

Lee K H, Cho S P, Oh T I and Woo E J 2006 Constant current source for a multi-frequency EIT system with $10 \mathrm{~Hz}$ to $500 \mathrm{kHz}$ operating frequency IFMBE World Cong. on Medical Physics and Biomedical Engineering (Seoul, Korea) p 5179

Lingwood B E, Dunster K R, Colditz P B and Ward L C 2002 Noninvasive measurement of cerebral bioimpedance for detection of cerebral edema in the neonatal piglet Brain Res. 945 97-105

Olsson T and Victorin L 1970 Transthoracic impedance, with special reference to newborn infants and the ratio air-to-fluid in the lungs Acta Paediatr. Scand. Suppl. 207 (Suppl. 207)

Ross A S, Saulnier G J, Newell J C and Isaacson D 2003 Current source design for electrical impedance tomography Physiol. Meas. 24 509-16

Seoane F, Bragos R and Lindecrantz K 2006 Current source for multifrequency broadband electrical bioimpedance spectroscopy systems. A novel approach Conf. Proc. IEEE Eng. Med. Biol. Soc. 1 5121-5

Seoane F, Lindecrantz K, Olsson T, Kjellmer I, Flisberg A and Bågenholm R 2005 Spectroscopy study of the dynamics of the transencephalic electrical impedance in the perinatal brain during hypoxia Physiol. Meas. 26 849-63 
Sheingold D (ed) 1966 Applications Manual for Computing Amplifiers for Modeling, Measuring, Manipulating \& Much Else (Boston, MA: Philbrick Researchers)

Skourou C, Hoopes P J, Strawbridge R R and Paulsen K D 2004 Feasibility studies of electrical impedance spectroscopy for early tumor detection in rats Physiol. Meas. 25 335-46
Skourou C, Rohr A, Hoopes P J and Paulsen K D 2007 In vivo EIS characterization of tumour tissue properties is dominated by excess extracellular fluid Phys. Med. Biol. 52 347-63

Van Kreel B K 2001 Multi-frequency bioimpedance measurements of children in intensive care Med. Biol. Eng. Comput. 39 551-7 